\title{
Multimarker Approach to Risk Stratification for Long-Term Mortality in Patients on Chronic Hemodialysis
}

\author{
Junnichi Ishii, MD; Hiroshi Takahashi, BSc; Fumihiko Kitagawa; Atsuhiro Kuno; \\ Ryuunosuke Okuyama, MD; Hideki Kawai, MD; Takashi Muramatsu, MD; Hiroyuki Naruse, MD; \\ Sadako Motoyama, MD; Shigeru Matsui, MD; Midori Hasegawa, MD; Toru Aoyama, MD; \\ Daisuke Kamoi, MD; Hirotake Kasuga, MD; Hideo Izawa, MD; Yukio Ozaki, MD; Yukio Yuzawa, MD
}

\begin{abstract}
Background: We prospectively investigated the prognostic value of the combined use of cardiac troponin $\mathrm{T}$ (TnT), B-type natriuretic peptide (BNP), and high-sensitivity C-reactive protein (CRP) for long-term mortality in hemodialysis (HD) patients.
\end{abstract}

Methods and Results: Baseline measurements of TnT, BNP, and CRP were performed in 516 patients on chronic HD. Patients were followed up for 10 years. Using the Cox multivariate model with these 3 biomarkers as variables categorized into tertiles for mortality, a simplified score was obtained by underscoring individual biomarkers based on the adjusted hazard ratio (HR). The multimarker score was defined as the sum of these points. TnT, BNP, and CRP levels were individually independent predictors for mortality $(\mathrm{P}<0.05)$. Among low-risk (multimarker score $<4)$, intermediate-risk (multimarker score 4-7), and high-risk (multimarker score $\geq 7$ ) groups, 10-year survival rates were 83.3\%, 54.3\%, and 27.2\% ( $<<0.0001)$, respectively. After adjusting for other confounders, the multimarker score had strong predictive power for mortality (HR: 4.26; $\mathrm{P}<0.0001$ for high-risk vs. low-risk group). Furthermore, adding the multimarker score to a baseline model with established risk factors improved the $\mathrm{C}$-index $(\mathrm{P}<0.01)$, net reclassification improvement $(P<0.0001)$, and integrated discrimination improvement $(P<0.0001)$ greater than that of any single biomarker or baseline model alone.

Conclusions: The multimarker approach (ie, simultaneous assessment of TnT, BNP, and CRP, which individually independently predict prognosis) may improve the prediction of long-term mortality in HD patients. (Circ $\mathrm{J} 2015$; 79: 656-663)

Key Words: B-type natriuretic peptide; Cardiac troponin T; Hemodialysis; High-sensitivity C-reactive protein; Mortality

$\mathbf{M}$ ortality rates for end-stage renal disease (ESRD) patients remains extraordinarily high, particularly because they often have concomitant cardiovascular disease. ${ }^{1}$ Thus, early and accurate risk stratification for mortality is critical in facilitating more aggressive and focalized treatment. ESRD has a unique risk factor profile, and predictive models developed in the general population therefore cannot be applied to ESRD patients. ${ }^{2}$ The use of traditional risk factors and simple clinical information does not fully explain the risk of death in patients on hemodialysis (HD). ${ }^{3}$ Moreover, a single biomarker may not adequately assess the risk. $^{2}$ Therefore, a multimarker approach (ie, simultaneous assessment of multiple biomarkers with individually different pathophysiological pathways) may refine risk stratification in HD patients. However, the role of the multimarker approach requires further evaluation in this population. ${ }^{2}$

\section{Editorial p 522}

Elevated levels of cardiac troponin T (TnT), B-type natriuretic peptide (BNP), and high-sensitivity C-reactive protein (CRP) in HD patients are associated with increased mortality, and are good prognostic markers. ${ }^{4-11}$ A meta-analysis concluded that an increase in TnT level conferred a 3-fold increase in

Received August 17, 2014; revised manuscript received December 3, 2014; accepted December 4, 2014; released online January 16, 2015 Time for primary review: 37 days

Department of Joint Research Laboratory of Clinical Medicine (J.I., F.K., A.K., H.N.), Division of Statistics (H.T.), Department of Cardiology (R.O., H. Kawai, T.M., S. Motoyama, S. Matsui, Y.O.), Department of Nephrology (M.H., Y.Y.), Fujita Health University School of Medicine, Toyoake; Cardiovascular Center (T.A., D.K.), Department of Nephrology (H. Kasuga), Nagoya Kyoritsu Hospital, Nagoya; and Department of Cardiology, Banbuntane Houtokukai Hospital, Nagoya (H.I.), Japan

Mailing address: Junnichi Ishii, MD, Department of Joint Research Laboratory of Clinical Medicine, Fujita Health University School of Medicine, 1-98 Dengakugakubo, Kutsukake-cho, Toyoake 470-1192, Japan. E-mail: jishii@ fujita-hu.ac.jp

ISSN-1346-9843 doi:10.1253/circj.CJ-14-0915

All rights are reserved to the Japanese Circulation Society. For permissions, please e-mail: cj@j-circ.or.jp 
the mortality risk in ESRD patients. ${ }^{12}$ However, the clinical interpretation of elevated levels of cardiac troponin I (TnI) has remained inconclusive because the TnI assay is not standardized. ${ }^{12,13}$ In addition, a recent meta-analysis provided evidence that elevated BNP and N-terminal pro-BNP (NT-proBNP) levels predicted a 4 -fold increase in the risk of mortality, and suggested that a given proportional increment in each biomarker was similarly associated with an increased risk of mortality. ${ }^{14}$ Furthermore, a large international study of HD patients demonstrated that CRP improved the 1-year mortality prediction. ${ }^{15}$ The 3 biomarkers (TnT, BNP, and CRP) individually assess different pathophysiological pathways (ie, myocardial injury, left ventricular wall stress, and inflammation, respectively); moreover, they are readily measured, easily accessible, relatively inexpensive, and reproducible. ${ }^{16} \mathrm{We}$ prospectively investigated whether the combined use of these biomarkers could improve the prediction of long-term mortality in HD patients.

\section{Methods}

\section{Study Population}

This prospective cohort study was conducted at Nagoya Kyoritsu Hospital (Nagoya, Japan). We enrolled 516 Japanese outpatients who were stable and underwent regular HD therapy for at least 3 months in April 2000. Patients with acute renal failure, active inflammatory diseases or malignancies were excluded. Diabetes was defined as a history or presence of diabetes and/or a fasting plasma glucose level $\geq 126 \mathrm{mg} / \mathrm{dl}$, hemoglobin A1c value $\geq 6.5 \%$, or the presence of diabetic retinopathy. Hypertension was defined as systolic blood pressure $\geq 140 \mathrm{mmHg}$ and/or diastolic blood pressure $\geq 90 \mathrm{mmHg}$, or a history of antihypertensive treatment. Dyslipidemia was defined as total cholesterol level $\geq 220 \mathrm{mg} / \mathrm{dl}$ or a history of lipid-lowering therapy. Smoking history was defined either as a current smoker or as having discontinued cigarette use within 6 months prior to study.

\section{Measurements of TnT, BNP, and CRP}

Blood samples for baseline measurements of serum TnT, plasma BNP, and serum CRP were obtained from patients before initiating individual HD sessions and before heparin administration, and were assayed within 1 week after sampling. Blood samples for measuring serum TnT and CRP were centrifuged at $4^{\circ} \mathrm{C}$ for $15 \mathrm{~min}$ at $1,000 \mathrm{~g}$, and stored at $-70^{\circ} \mathrm{C}$ until assayed. The third-generation Enzymun Test Troponin $\mathrm{T}$ assay was used, based on a prototype of the electrochemiluminescence-based Elecsys system (Roche Diagnostics K.K., Tokyo, Japan). The manufacturer's stated detection limit is $<0.01 \mathrm{ng} / \mathrm{ml}$. The lowest concentration to attain a coefficient of variation $<10 \%$ is $0.03 \mathrm{ng} / \mathrm{ml}$. Serum CRP levels were measured using a latex-enhanced high-sensitivity CRP immunoassay (Siemens Healthcare Diagnostics K.K., Tokyo, Japan) with an extended measurement of $0.175-200 \mathrm{mg} / \mathrm{L}$. A normal cutoff of $<1.0 \mathrm{mg} / \mathrm{L}$, based on American Heart Association guidelines, ${ }^{17}$ was used in the risk assessment.

Blood samples for measuring plasma BNP were collected in chilled tubes containing ethylenediaminetetraacetic acid, disodium salt, and aprotinin $(500 \mathrm{IU} / \mathrm{ml})$. The plasma was separated by centrifugation at $4^{\circ} \mathrm{C}$ for $15 \mathrm{~min}$ at $1,000 \mathrm{~g}$ and then stored at $-70^{\circ} \mathrm{C}$ until analysis. BNP concentrations were measured using a commercial radioimmunoassay for human BNP (Shiono RIA BNP assay; Shionogi Co, Ltd, Osaka, Japan). The manufacturer's stated detection limit and upper limit of the reference interval were $<2 \mathrm{pg} / \mathrm{ml}$ and $18.4 \mathrm{pg} / \mathrm{ml}$, respectively.

\section{Follow-up Study}

Patients were prospectively followed up until June 2010. The primary endpoint was all-cause death. The secondary endpoint was cardiovascular death (eg, death from heart failure, myocardial infarction, arrhythmia, sudden death, or stroke). Data for the endpoints were obtained from hospital charts and through telephone interviews with patients. The telephone interviews were conducted by trained reviewers who were blinded to the patients' marker levels. When patients were transferred to other hospitals or received renal transplantation, their follow-up was censored at those time points.

\section{Ethics}

The study protocol was approved by the institutional ethics committee, and was conducted in accordance with the Declaration of Helsinki. The physicians were given written informed consent from each patient.

\section{Statistical Analysis}

Statistical analyses were performed using SAS 6.10 software (SAS Institute, Cary, NC, USA). Normally distributed variables are expressed as mean value \pm standard deviation, and nonparametric data are presented as median and interquartile range. The lower detection limit of the TnT assay was $<0.01 \mathrm{ng} / \mathrm{ml}$. TnT levels $<0.01 \mathrm{ng} / \mathrm{ml}$ were therefore assigned a value of $0.005 \mathrm{ng} / \mathrm{ml}$ for calculations. Intergroup differences were evaluated using one-way analysis of variance or KruskalWallis test for continuous variables and chi-square test for categorical variables. Intergroup differences in survival were examined using the Kaplan-Meier method and compared using log-rank test. Hazard ratio (HR) and $95 \%$ confidence intervals (CI) were calculated for each factor using Cox proportional hazards analysis. All baseline variables with $\mathrm{P}<0.05$ by univariate analysis were entered into a Cox multivariate model to determine independent predictors for the endpoints.

The cutoff values of the biomarkers were based on tertiles of the individual biomarkers, and a multivariate Cox analysis including these 3 biomarkers for all-cause mortality was performed. Based on the analyzed model, a simplified score was obtained by underscoring individual biomarkers proportional to the adjusted HR. The multimarker score was then defined as the sum of these points, with higher points indicating a higher mortality risk.

To assess whether the accuracy of predicting mortality would improve after adding the multimarker score or each single biomarker into a baseline model with established risk factors (ie, sex, age, diabetes, hypertension, dyslipidemia, smoking status, body mass index, previous cardiovascular diseases, hemoglobin, and albumin), we calculated the C-index, net reclassification improvement (NRI), and integrated discrimination improvement (IDI). The C-index is defined as the area under receiver-operating characteristic curves between individual predictive probabilities for mortality and the incidence of mortality, and was compared for the baseline model and enriched models containing the established risk factors plus the multimarker score or each single biomarker, respectively. ${ }^{18} \mathrm{NRI}$ indicates relatively how many patients improved their predicted probability for mortality, and IDI represents the average improvement in predicted probability for mortality after adding variables into the baseline model. ${ }^{19}$ Differences were considered statistically significant at $\mathrm{P}<0.05$. 


\begin{tabular}{|c|c|c|c|c|c|}
\hline & \multirow{2}{*}{$\begin{array}{c}\text { All patients } \\
(n=516)\end{array}$} & \multicolumn{3}{|c|}{ Multimarker score } & \multirow[b]{2}{*}{$P$ value } \\
\hline & & $\begin{array}{c}\text { Low-risk group }<4 \\
(n=192)\end{array}$ & $\begin{array}{l}\text { Intermediate-risk } \\
\text { group 4-7 }(n=188)\end{array}$ & $\begin{array}{c}\text { High-risk group } \geq 7 \\
(n=136)\end{array}$ & \\
\hline Male & 309 (59.9) & $104(54.2)$ & $117(62.2)$ & $88(64.7)$ & 0.14 \\
\hline Age (years) & $60 \pm 12$ & $56 \pm 11$ & $62 \pm 10$ & $65 \pm 12$ & $<0.0001$ \\
\hline Duration of hemodialysis (years) & $5.5(2.0-11.4)$ & $4.9(1.6-9.6)$ & $5.6(2.4-13.3)$ & $5.3(2.0-9.9)$ & 0.15 \\
\hline Diabetes & $140(27.1)$ & $47(24.5)$ & $50(26.6)$ & $43(31.6)$ & 0.24 \\
\hline Hypertension & $344(66.7)$ & $132(68.8)$ & $130(69.2)$ & $82(60.3)$ & 0.22 \\
\hline Dyslipidemia & $78(15.1)$ & $31(16.1)$ & $27(14.4)$ & $20(14.7)$ & 0.28 \\
\hline Smoking & $149(28.8)$ & $58(30.4)$ & $57(30.3)$ & $34(25.0)$ & 0.63 \\
\hline BMI $\left(\mathrm{kg} / \mathrm{m}^{2}\right)$ & $20.6 \pm 2.9$ & $21.1 \pm 2.9$ & $20.6 \pm 2.7$ & $19.7 \pm 3.0$ & 0.0015 \\
\hline Previous heart failure & $54(10.5)$ & $13(6.8)$ & $21(11.2)$ & $20(14.7)$ & 0.092 \\
\hline PCAD & $172(33.3)$ & $40(20.8)$ & $66(35.1)$ & $66(48.5)$ & $<0.0001$ \\
\hline Previous stroke & $26(5.0)$ & $1(0.6)$ & $16(8.5)$ & $9(6.7)$ & 0.0017 \\
\hline PPAD & $22(4.3)$ & $1(0.5)$ & $9(4.8)$ & $12(8.8)$ & 0.0010 \\
\hline $\mathrm{TnT}(\mathrm{ng} / \mathrm{ml})$ & $0.07(0.04-0.13)$ & $0.03(0.01-0.05)$ & $0.09(0.06-0.12)$ & $0.14(0.09-0.19)$ & $<0.0001$ \\
\hline BNP (pg/ml) & $268(147-627)$ & $166(91-262)$ & $272(159-569)$ & $717(482-1,267)$ & $<0.0001$ \\
\hline CRP (mg/L) & $2.8(1.0-11.8)$ & $1.0(0.7-3.1)$ & $2.6(1.0-8.5)$ & $12.5(4.0-30.6)$ & $<0.0001$ \\
\hline Hemoglobin (g/dl) & $10.4 \pm 1.4$ & $10.8 \pm 1.3$ & $10.2 \pm 1.4$ & $10.0 \pm 1.5$ & $<0.0001$ \\
\hline Albumin (g/dl) & $3.6 \pm 0.4$ & $3.7 \pm 0.3$ & $3.6 \pm 0.4$ & $3.5 \pm 0.4$ & $<0.0001$ \\
\hline
\end{tabular}

Data are present as mean \pm standard deviation, number of patients (percentage), or median (interquartile range). BMI, body mass index; BNP B-type natriuretic peptide; CRP, C-reactive protein; PCAD, previous coronary artery disease; PPAD, previous peripheral artery disease; TnT, cardiac troponin $\mathrm{T}$.

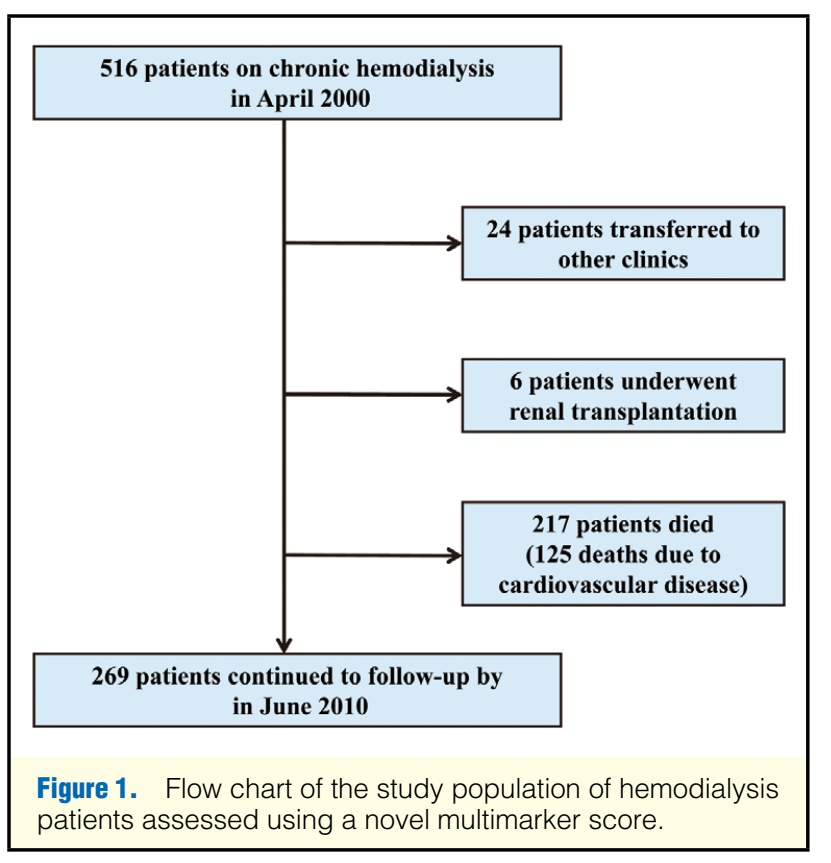

\section{Results}

\section{Baseline Characteristics and Prognostic Value}

Characteristics of the study population are listed in Table 1. A flow diagram for the study population is shown in Figure 1. Of the total patients, 24 were transferred to other hospitals and 6 received a renal transplantation; their follow-up was censored at these time points. During an average follow-up period of $81 \pm 41$ months, 217 patients $(42.1 \%)$ died, 125 (24.2\%) from cardiovascular disease. In the Cox multivariate analysis in- cluding all baseline variables with $\mathrm{P}<0.05$ by univariate analysis (ie, the 3 biomarkers, age, diabetes, body mass index, previous coronary artery disease, hemoglobin, and albumin), TnT, BNP, and CRP levels were individually independent predictors for all-cause mortality (HR: $1.21,95 \%$ CI: 1.10-1.31, $\mathrm{P}=0.0003$; HR: 1.02 , 95\% CI: 1.01-1.04, $\mathrm{P}=0.018$; and HR: 1.09, 95\% CI: 1.03-1.14, $\mathrm{P}=0.0015$, respectively) (Table 2).

Patients were divided into tertiles on the basis of TnT levels (lowest, $\leq 0.05$; middle, $0.05-0.10$; and highest, $>0.10 \mathrm{ng} / \mathrm{ml}$ ), BNP levels (lowest, $\leq 185$; middle, 185-464; and highest, $>464 \mathrm{pg} / \mathrm{ml}$ ), and CRP levels (lowest, $\leq 1.3$; middle, 1.3-7.0; and highest, $>7.0 \mathrm{mg} / \mathrm{L}$ ) (Table 3 ). The 10-year Kaplan-Meier survival rates in the lowest, middle, and highest tertiles were $89.4 \%, 53.2 \%$, and $36.8 \%$, respectively, according to TnT levels $(\mathrm{P}<0.0001) ; 73.8 \%, 61.8 \%$, and $38.0 \%$, respectively, according to BNP levels ( $\mathrm{P}<0.0001)$; and $79.4 \%, 61.7 \%$, and $32.7 \%$, respectively, according to CRP levels $(\mathrm{P}<0.0001)$ (Figure 2).

\section{Prognostic Value of Multimarker Score}

The multivariate Cox analysis including TnT, BNP, and CRP as variables categorized into tertiles for all-cause mortality is shown in Table 3 . The rules for the multimarker score were: (1) the score for the nonsignificant subgroup (BNP, from $>185$ to $\leq 464 \mathrm{pg} / \mathrm{ml}$ ) was set to be zero, (2) the score for the other subgroups was set to the corresponding HR rounded up to an integer (Table 3), and (3) the multimarker score was defined as the sum of the scores.

According to the multimarker score, patients were classified into low-risk ( $<4$ points), intermediate-risk (4-7 points), and high-risk ( $\geq 7$ points) groups. Patients in the high-risk group were older, had higher TnT, BNP, and CRP levels, and lower body mass index, hemoglobin, and albumin levels. In addition, a history of coronary artery disease, stroke, or peripheral artery disease was more frequent in patients in the high-risk group than those in low-risk group (Table 1). 
Table 2. Predictors of All-Cause Mortality in the Study Population of Hemodialysis Patients

TnT (per $0.1 \mathrm{ng} / \mathrm{ml}$ increment)

BNP (per $100 \mathrm{pg} / \mathrm{ml}$ increment)

CRP (per $1 \mathrm{mg} / \mathrm{L}$ increment)

Age (per 1 year increment)

Diabetes

BMI (per $1 \mathrm{~kg} / \mathrm{m}^{2}$ increment)

PCAD

Hemoglobin (per $1 \mathrm{~g} / \mathrm{dl}$ increment)

Albumin (per $1 \mathrm{~g} / \mathrm{dl}$ increment)

\begin{tabular}{ccccc}
\multicolumn{2}{c}{ Univariate } & & \multicolumn{2}{c}{ Multivariate } \\
\cline { 1 - 2 } \cline { 5 - 6 } HR (95\% Cl) & P value & & HR (95\% Cl) & P value \\
$1.23(1.15-1.32)$ & $<0.0001$ & & $1.21(1.10-1.31)$ & 0.0003 \\
$1.05(1.04-1.06)$ & $<0.0001$ & & $1.02(1.01-1.04)$ & 0.018 \\
$1.08(1.05-1.11)$ & $<0.0001$ & & $1.09(1.03-1.14)$ & 0.0015 \\
$1.06(1.05-1.08)$ & $<0.0001$ & & $1.05(1.03-1.07)$ & $<0.0001$ \\
$1.45(1.07-1.94)$ & 0.016 & & $1.32(0.93-1.87)$ & 0.11 \\
$0.88(0.82-0.94)$ & 0.0001 & & $0.94(0.87-1.01)$ & 0.10 \\
$1.74(1.31-2.79)$ & 0.0001 & & $1.27(0.87-1.88)$ & 0.63 \\
$0.71(0.64-0.79)$ & $<0.0001$ & & $0.87(0.76-0.98)$ & 0.027 \\
$0.47(0.42-0.57)$ & $<0.0001$ & & $0.45(0.28-0.75)$ & 0.0021 \\
\hline
\end{tabular}

Multivariate model included TnT, BNP, CRP, age, diabetes, BMI, PCAD, hemoglobin, and albumin as baseline variables with $\mathrm{P}<0.05$ by univariate analysis. $\mathrm{Cl}$, confidence interval; $\mathrm{HR}$, hazard ratio. Other abbreviations as in Table 1.

\begin{tabular}{|c|c|c|c|c|c|}
\hline \multirow{2}{*}{ Biomarker } & \multicolumn{2}{|c|}{ Individual } & \multicolumn{2}{|c|}{ Combined } & \multirow{2}{*}{ Add to score } \\
\hline & HR $(95 \% \mathrm{Cl})$ & $P$ value & HR $(95 \% \mathrm{Cl})$ & $P$ value & \\
\hline \multicolumn{6}{|l|}{ TnT (ng/ml) } \\
\hline$\leq 0.05$ & Ref. & & Ref. & & 0 \\
\hline$>0.05$ to $\leq 0.10$ & $3.10(1.97-4.89)$ & $<0.0001$ & $2.62(1.64-4.17)$ & $<0.0001$ & +3 \\
\hline$>0.10$ & $5.44(3.51-8.43)$ & $<0.0001$ & $3.57(2.24-5.69)$ & $<0.0001$ & +4 \\
\hline \multicolumn{6}{|l|}{ BNP (pg/ml) } \\
\hline$\leq 185$ & Ref. & & Ref. & & 0 \\
\hline$>185$ to $\leq 464$ & $1.64(1.10-2.43)$ & 0.015 & $1.32(0.88-1.99)$ & 0.18 & 0 \\
\hline$>464$ & $3.12(2.16-4.50)$ & $<0.0001$ & $1.99(1.35-2.94)$ & 0.0006 & +2 \\
\hline \multicolumn{6}{|l|}{ CRP (mg/L) } \\
\hline$\leq 1.3$ & Ref. & & Ref. & & 0 \\
\hline$>1.3$ to $\leq 7.0$ & $1.79(1.17-2.73)$ & 0.0069 & $1.65(1.07-2.52)$ & 0.022 & +2 \\
\hline$>7.0$ & $4.65(3.17-6.83)$ & $<0.0001$ & $3.31(2.22-4.91)$ & $<0.0001$ & +3 \\
\hline
\end{tabular}

Combined model included TnT, BNP, and CRP as variables categorized into tertiles. Ref., reference. Other abbreviations as in Tables 1,2.

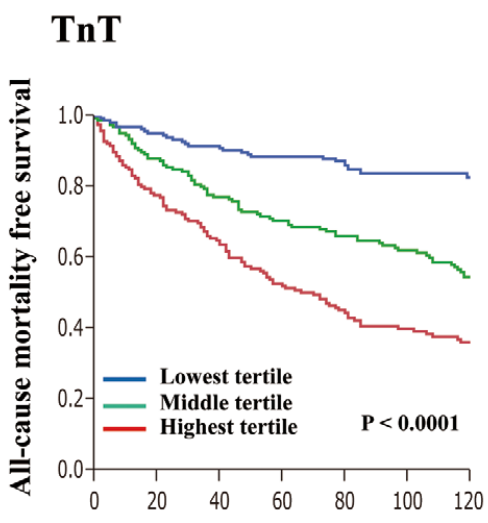

Follow-up period (months)

\section{BNP}

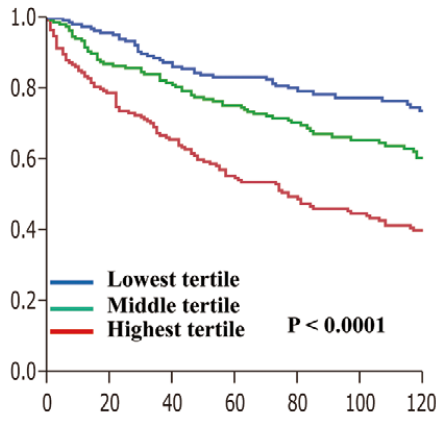

Follow-up period (months)

\section{CRP}



Follow-up period (months)

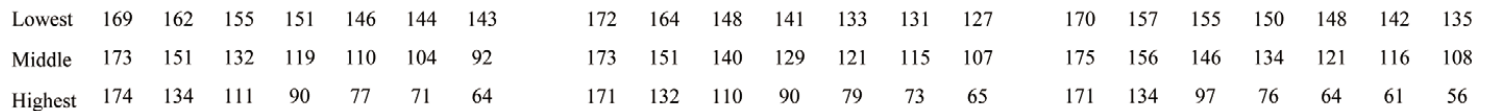

Figure 2. Kaplan-Meier curves for all-cause mortality of the study population of hemodialysis patients based on tertiles of troponin $\mathrm{T}(\mathrm{TnT})$, B-type natriuretic peptide (BNP), and C-reactive protein (CRP). 


\section{A}

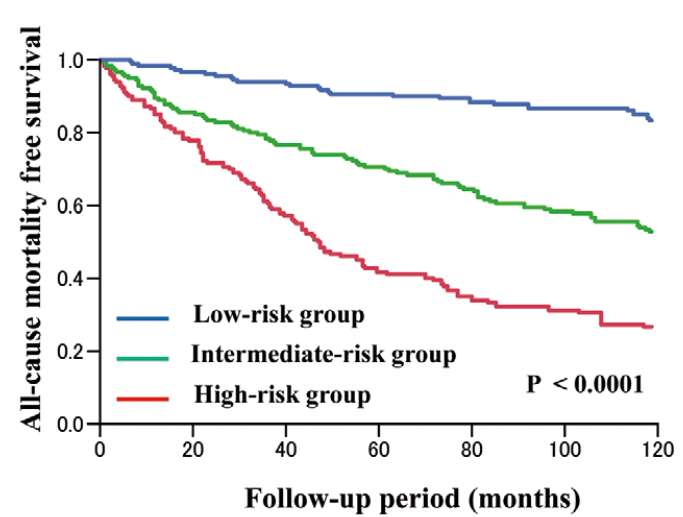

B

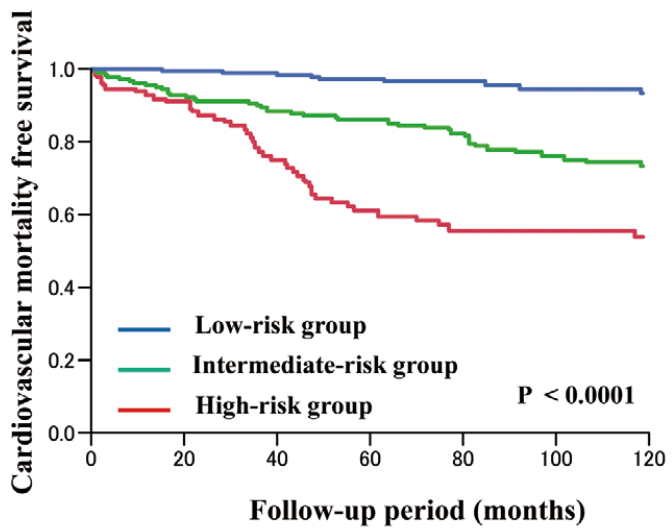

Follow-up period (months)

$\begin{array}{lccccccc}\text { Low-risk group } & 192 & 184 & 178 & 171 & 167 & 165 & 160 \\ \text { Intermediate-risk group } & 188 & 160 & 144 & 133 & 120 & 111 & 102 \\ \text { High-risk group } & 136 & 103 & 76 & 56 & 46 & 43 & 37\end{array}$

Figure 3. Kaplan-Meier curves for (A) all-cause and (B) cardiovascular mortality based on a multimarker score in the low-risk, intermediate-risk and high-risk groups in the study population of hemodialysis patients.

\begin{tabular}{|c|c|c|c|c|}
\hline & \multicolumn{2}{|c|}{ All-cause mortality } & \multicolumn{2}{|c|}{ Cardiovascular mortality } \\
\hline & HR $(95 \% \mathrm{Cl})$ & $P$ value & HR $(95 \% \mathrm{Cl})$ & $P$ value \\
\hline Mmultimarker score & & $<0.0001^{*}$ & & $<0.0001^{*}$ \\
\hline Low-risk (<4) & Ref. & & Ref. & \\
\hline Intermediate risk (4-7) & $2.30(1.42-3.71)$ & 0.0007 & $3.47(1.56-7.69)$ & 0.0022 \\
\hline High risk $(\geq 7)$ & $4.26(2.59-7.00)$ & $<0.0001$ & $7.07(3.15-15.9)$ & $<0.0001$ \\
\hline Age (per 1 year increment) & $1.04(1.03-1.06)$ & $<0.0001$ & $1.03(1.01-1.05)$ & 0.0094 \\
\hline Diabetes & $1.43(1.03-2.01)$ & 0.033 & $1.91(1.19-3.07)$ & 0.0075 \\
\hline BMI (per $1 \mathrm{~kg} / \mathrm{m}^{2}$ increment) & $0.95(0.89-1.02)$ & 0.13 & $0.95(0.86-1.05)$ & 0.32 \\
\hline PCAD & $1.08(0.75-1.54)$ & 0.68 & $1.44(0.85-2.43)$ & 0.18 \\
\hline Hemoglobin (per $1 \mathrm{~g} / \mathrm{dl}$ increment) & $0.87(0.78-0.98)$ & 0.018 & $0.95(0.80-1.12)$ & 0.24 \\
\hline Albumin (per $1 \mathrm{~g} / \mathrm{dl}$ increment) & $0.46(0.29-0.74)$ & 0.0013 & $0.65(0.31-1.35)$ & 0.54 \\
\hline
\end{tabular}

${ }^{*} \mathrm{P}$ value for trend. Adjustment for age, diabetes, BMI, PCAD, hemoglobin, and albumin as baseline variables with $\mathrm{P}<0.05$ by univariate analysis. Abbreviations as in Tables $1-3$.

The Kaplan-Meier 10-year survival rates for all-cause and cardiovascular mortality were $83.3 \%$ and $93.5 \%$, respectively, in the low-risk group, $54.3 \%$ and $73.3 \%$, respectively, in the intermediate-risk group, and $27.2 \%$ and $54.0 \%$, respectively, in the high-risk group (all $\mathrm{P}<0.0001$ ) (Figure 3).

In the multivariate Cox analyses including all baseline variables with $\mathrm{P}<0.05$ by univariate analysis, the multimarker score had strong predictive power for all-cause mortality (HR: 4.26, 95\% CI: 2.59-7.00, $\mathrm{P}<0.0001$ for high-risk vs. low-risk group) and cardiovascular mortality (HR: 7.07, 95\% CI: 3.15-15.9, $\mathrm{P}<0.0001$ for high-risk vs. low-risk group) (Table 4). In addition to the multimarker score, age, diabetes, hemoglobin, and albumin remained significantly associated with all-cause mortality. Age and diabetes remained significantly associated with cardiovascular mortality.

\section{Discrimination and Reclassification of Multimarker Score}

Adding the multimarker score to a baseline model with established risk factors improved the prediction of all-cause mortality beyond that of any single biomarker (all $\mathrm{P}<0.01$ ) or baseline model alone $(\mathrm{P}<0.0001)$, as shown by the significant increase in the $\mathrm{C}$-index (Table 5). Reclassification of patients who died or were alive at follow-up is presented by NRI. The addition of the multimarker score significantly (all $\mathrm{P}<0.0001$ ) improved the reclassification of patients beyond that of any single biomarker or baseline model alone. In addition, IDI improved significantly (all $\mathrm{P}<0.0001$ ) after adding the multimarker score, beyond that of any single biomarker or baseline model alone. Similar results were seen for cardiovascular mortality (Table 5). 


\begin{tabular}{|c|c|c|c|c|c|c|}
\hline & C-index $(95 \% \mathrm{Cl})$ & $P$ value & NRI & $P$ value & IDI & $P$ value \\
\hline \multicolumn{7}{|l|}{ All-cause mortality } \\
\hline Established risk factors & $0.745(0.702-0.788)$ & Ref. & & Ref. & & Ref. \\
\hline Established risk factors+TnT & $0.786(0.746-0.826)$ & 0.0006 & 0.429 & $<0.0001$ & 0.069 & $<0.0001$ \\
\hline Established risk factors+BNP & $0.785(0.745-0.825)$ & 0.0011 & 0.286 & 0.0010 & 0.053 & $<0.0001$ \\
\hline Established risk factors+CRP & $0.773(0.732-0.814)$ & 0.0029 & 0.541 & $<0.0001$ & 0.036 & $<0.0001$ \\
\hline Established risk factors+multimarker score & $0.828(0.792-0.865)$ & $<0.0001$ & 0.664 & $<0.0001$ & 0.135 & $<0.0001$ \\
\hline \multicolumn{7}{|l|}{ Cardiovascular mortality } \\
\hline Established risk factors & $0.705(0.645-0.765)$ & Ref. & & Ref. & & Ref. \\
\hline Established risk factors+TnT & $0.740(0.685-0.796)$ & 0.010 & 0.312 & 0.0043 & 0.013 & 0.028 \\
\hline Established risk factors+BNP & $0.725(0.667-0.783)$ & 0.049 & 0.212 & 0.038 & 0.015 & 0.051 \\
\hline Established risk factors+CRP & $0.723(0.666-0.781)$ & 0.030 & 0.198 & 0.047 & 0.005 & 0.091 \\
\hline Established risk factors+multimarker score & $0.778(0.731-0.824)$ & 0.0045 & 0.558 & $<0.0001$ & 0.066 & $<0.0001$ \\
\hline \multicolumn{7}{|l|}{ All-cause mortality } \\
\hline+ Multimarker score vs. $+\mathrm{TnT}$ & $0.043(0.017-0.068)^{\star}$ & 0.0009 & 0.64 & $<0.0001$ & 0.082 & $<0.0001$ \\
\hline +Multimarker score vs. +BNP & $0.043(0.017-0.069)^{\star}$ & 0.0013 & 0.517 & $<0.0001$ & 0.083 & $<0.0001$ \\
\hline +Multimarker score vs. +CRP & $0.055(0.024-0.086)^{\star}$ & 0.0005 & 0.575 & $<0.0001$ & 0.010 & $<0.0001$ \\
\hline \multicolumn{7}{|l|}{ Cardiovascular mortality } \\
\hline +Multimarker score vs. $+\mathrm{TnT}$ & $0.037(0.001-0.079)^{\star}$ & 0.049 & 0.537 & $<0.0001$ & 0.052 & $<0.0001$ \\
\hline +Multimarker score vs. +BNP & $0.053(0.008-0.097)^{\star}$ & 0.019 & 0.667 & $<0.0001$ & 0.051 & $<0.0001$ \\
\hline +Multimarker score vs. +CRP & $0.054(0.007-0.102)^{\star}$ & 0.025 & 0.547 & $<0.0001$ & 0.061 & $<0.0001$ \\
\hline
\end{tabular}

Established risk factors included sex, age, diabetes, hypertension, dyslipidemia, smoking status, BMI, previous cardiovascular diseases, hemoglobin, and albumin. *Estimated differences between 2 groups. Abbreviations as in Tables 1-3.

\section{Discussion}

This prospective study is, to the best of our knowledge, the first to demonstrate that TnT, BNP, and CRP each may be independent predictors of long-term mortality in patients on chronic HD, and that a multimarker approach (ie, simultaneous assessment of these 3 biomarkers, which individually reflect myocardial injury, left ventricular wall stress, and inflammation) may improve the predictive value for all-cause and cardiovascular mortality beyond any single biomarker, as demonstrated by C-index, NRI, and IDI. These findings support the usefulness of the multimarker approach as part of an algorithm for assessing long-term prognosis in HD patients. ${ }^{2}$

\section{Previous Multimarker Studies}

Cardiovascular disease is the leading cause of death in HD patients. ${ }^{1}$ However, the potential complementary roles of combining measurements of cardiovascular biomarkers such as TnT/TnI, BNP/NT-proBNP, and CRP require further evaluation. ${ }^{20-29}$ In fact, only a few studies with small populations have investigated the incremental predictive value of the simultaneous assessment of the 3 cardiovascular biomarkers in a single prognostic model. ${ }^{25-29}$ Furthermore, research thus far has generated conflicting results.

Recently, Bargnoux et $\mathrm{al}^{25}$ suggested that a combination of TnI, NT-proBNP, and CRP levels possibly improved risk assessment for short-term ( $<2$ years) mortality in $140 \mathrm{HD}$ patients. In their study, BNP and CRP were independent predictors of mortality but not TnI, probably because of the small study population. In addition, the prognostic ability of the addition of the 3 biomarkers to established risk factors was not assessed. Another study of 109 HD patients with a mean follow-up period of 926 days showed that both TnT and CRP levels were independent predictors of mortality, but NTproBNP was not; ${ }^{26}$ it also showed that the addition of TnT and
CRP significantly increased the C-index and IDI. Moreover, a study of $206 \mathrm{HD}$ patients with a median follow-up period of 28 months showed that a combination of TnI using a highsensitivity assay and BNP could provide additional prognostic information for mortality. ${ }^{27}$ Finally, in a study of 143 dialysis patients comparing the prognostic value of TnT, NT-proBNP, and CRP, NT-proBNP was the best predictor of outcome at the median follow-up of 30 months. ${ }^{28}$ However, TnT proved to be the best predictor of outcome at the median follow-up of 46.7 months,${ }^{29}$ suggesting that cardiac biomarkers had different prognostic abilities at different time points: NT-proBNP was a better predictor for early mortality and troponin for later mortality. Thus, we prospectively investigated the prognostic value of the combination of these 3 cardiovascular biomarkers in a relatively large study population $(n=516)$ with long-term (10 years) follow-up.

\section{Multimarker Score}

Cardiovascular disease in HD patients may be quite heterogeneous, ${ }^{30}$ and a single biomarker may not adequately assess the risk. $^{2}$ TnT, BNP, and CRP levels individually reflect a unique pathophysiological pathway in cardiovascular disease, and may identify different subpopulations at increased risk of mortality. Thus, it is not surprising that simultaneous assessment of these 3 biomarkers yielded independent and complementary prognostic information. These biomarkers are readily measured, easily accessible, relatively inexpensive, and reproducible with high sensitivity and specificity. ${ }^{16}$ The multimarker score is simple, has strong discriminative capacity, can accurately identify patients at low, intermediate, and high risk for all-cause and cardiovascular mortality, and may be useful in clinical practice. Furthermore, the addition of the multimarker score may substantially refine risk stratification models for long-term mortality. When confirmed by other independent cohorts, using standard, widely available assays and a 
simple scoring system, a multimarker score may be used as a part of an algorithm for assessing the long-term prognosis of HD patients.

In the present study, we focused on prognostic assessment of the combined use of 3 cardiovascular biomarkers: TnT, BNP, and CRP. Thus, we devised a multimarker score from these cardiovascular biomarkers. Age and the levels of hemoglobin and albumin, as well as the 3 cardiovascular biomarkers, were significantly associated with all-cause mortality. Thus, age, hemoglobin, and albumin might facilitate risk stratification when used in conjunction with the 3 cardiovascular biomarkers. This notion warrants testing in a future trial involving a large study population. We also assigned a different score to each degree of severity among the different cardiovascular biomarkers. The proposed scoring method is not widely accepted, although there are studies involving a similar method for patients with acute coronary syndrome. ${ }^{31}$ When all scores were set uniformly as 0,1 , or 2 in every degree of severity, C-indices of this scoring method for predicting allcause mortality ( 0.825 and 0.828 , respectively) and cardiovascular mortality ( 0.773 and 0.778 , respectively) were similar to the current scoring method (data not shown). Further research is necessary to develop an optimal scoring method.

\section{High-Sensitivity Troponin Assays}

A more sensitive formation assay for TnT or TnI, not available at the time of this study, can now be used. ${ }^{32-34}$ Re-evaluation is needed to determine the prognostic abilities of the combination of TnT or TnI using this high-sensitivity assay, BNP/ NT-proBNP, and CRP. In fact, a recent study, using this assay, found the TnT level to be a nonsignificantly more powerful predictor of mortality than was previously observed;29 however, the increase in the area under the curve of TnT with this high-sensitivity assay ( 0.760 vs. 0.746$)$ was very small and may not be clinically relevant for the overall prediction.

\section{Study Limitations}

First, this study had a single-center design. Larger multicenter studies are warranted to corroborate our findings. Second, all enrolled patients in the study were Japanese; Japanese patients are reported to have a better prognosis than corresponding patients in the United States and Europe ${ }^{35}$ because the prevalence of subclinical atherosclerosis, coronary disease mortality, and the risk of coronary calcification are lower in Japanese patients, ${ }^{36,37}$ and a lower prevalence of inflammation has been reported in dialysis patients in Asian countries, such as Japan and Korea (probably because of genetic factors and cultural habits such as food intake). ${ }^{15,38,39}$ These differences should be considered when interpreting the results. Third, we only measured biomarkers at the time of enrollment. Thus, we did not evaluate whether these biomarkers can also act as monitoring markers, and whether their improvement affects outcomes. Such analysis is necessary in subsequent studies. In addition, we did not evaluate precise therapeutic interventions; therefore, we did not have data regarding the effectiveness of medications for decreasing these biomarker levels and improving prognosis. Further studies are necessary to determine whether a treatment policy based on these biomarker levels reduces the high risk of mortality in HD patients. Fourth, we acknowledge the limits imposed by the absence of echocardiographic data. Echocardiography is recommended in current guidelines as a fundamental tool for profiling cardiovascular disease in patients with ESRD. ${ }^{40}$ However, echocardiography is often a limited resource and may not be routinely available. ${ }^{2}$ Several studies have suggested that TnT (or TnI) and BNP (or
NT-proBNP) may be potential predictors of mortality independent of left ventricular mass and ejection fraction, and may add prognostic information for adverse clinical outcomes to data from echocardiography. ${ }^{5,7,8,21,23}$ Finally, we did not have a validation data set.

\section{Conclusions}

Our data suggest that TnT, BNP, and CRP levels, individually, are independent predictors of long-term mortality in ESRD patients. A multimarker approach that incorporates these 3 biomarkers may substantially improve the prediction of allcause and cardiovascular mortality of HD patients.

\section{Disclosures}

Funders: None.

\section{References}

1. Nakai S, Suzuki K, Masakane I, Wada A, Itami N, Ogata S, et al. Overview of regular dialysis treatment in Japan (as of 31 December 2008). Ther Apher Dial 2010; 14: 505-540.

2. Ortiz A, Massy ZA, Fliser D, Lindholm B, Wiecek A, MartínezCastelao A, et al. Clinical usefulness of novel prognostic biomarkers in patients on hemodialysis. Nat Rev Nephrol 2011; 8: 141-150.

3. Zoccali C, Tripepi G, Mallamaci F. Predictors of cardiovascular death in ESRD. Semin Nephrol 2005; 25: 358-362.

4. Ishii J, Nomura M, Okuma T, Minagawa T, Naruse H, Mori Y, et al. Risk stratification using serum concentrations of cardiac troponin $\mathrm{T}$ in patients with end-stage renal disease on chronic maintenance dialysis. Clin Chim Acta 2001; 312: 69-79.

5. Mallamaci F, Zoccali C, Parlongo S, Tripepi G, Benedetto FA, Cutrupi S, et al. Troponin is related to left ventricular mass and predicts all-cause and cardiovascular mortality in hemodialysis patients. Am J Kidney Dis 2002; 40: 68-75.

6. Apple FS, Murakami MM, Pearce LA, Herzog CA. Predictive value of cardiac troponin I and T for subsequent death in end-stage renal disease. Circulation 2002; 106: $2941-2945$.

7. Zoccali C, Mallamaci F, Benedetto FA, Tripepi G, Parlongo S, Cataliotti A, et al; Creed Investigators. Cardiac natriuretic peptides are related to left ventricular mass and function and predict mortality in dialysis patients. J Am Soc Nephrol 2001; 12: 1508-1515.

8. Wang AY, Lam CW, Yu CM, Chan IH, Zhang Y, Lui SF, et al. Nterminal pro-brain natriuretic peptide: An independent risk predictor of cardiovascular congestion, mortality, and adverse cardiovascular outcomes in chronic peritoneal dialysis patients. J Am Soc Nephrol 2007; 18: $321-330$

9. Winkler K, Wanner C, Drechsler C, Lilienthal J, März W, Krane V; German Diabetes and Dialysis Study Investigators. Change in Nterminal-pro-B-type-natriuretic-peptide and the risk of sudden death, stroke, myocardial infarction, and all-cause mortality in diabetic dialysis patients. Eur Heart J 2008; 29: 2092-2099.

10. Arici M, Walls J. End-stage renal disease, atherosclerosis, and cardiovascular mortality: Is C-reactive protein the missing link? Kidney Int 2001; 59: 407-414.

11. Takahashi H, Ishii H, Aoyama T, Kamoi D, Kasuga H, Ito Y, et al. Association of cardiac valvular calcifications and C-reactive protein with cardiovascular mortality in incident hemodialysis patients: A Japanese cohort study. Am J Kidney Dis 2013; 61: 254-261.

12. Khan NA, Hemmelgarn BR, Tonelli M, Thompson CR, Levin A. Prognostic value of troponin $\mathrm{T}$ and I among asymptomatic patients with end-stage renal disease: A meta-analysis. Circulation 2005; 112: $3088-3096$.

13. Needham DM, Shufelt KA, Tomlinson G, Scholey JW, Newton GE. Troponin I and T levels in renal failure patients without acute coronary syndrome: A systematic review of the literature. Can J Cardiol 2004; 20: $1212-1218$

14. Cheng YJ, Yao FJ, Liu LJ, Tang K, Lin XX, Li WJ, et al. B-type natriuretic peptide and prognosis of end-stage renal disease: A metaanalysis. PLoS One 2013; 8: e79302, doi:10.1371/journal.pone. 0079302.

15. Bazeley J, Bieber B, Li Y, de Sequera P, Combe C, Yamamoto H, et al. C-reactive protein and prediction of 1-year mortality in prevalent hemodialysis patients. Clin J Am Soc Nephrol 2011; 6: 2452-2461.

16. Wang AY, Wai-Kei Lam C. The diagnostic utility of cardiac biomarkers in dialysis patients. Semin Dial 2012; 25: 388-396. 
17. Ridker PM. Clinical application of C-reactive protein for cardiovascular disease detection and prevention. Circulation 2003; 107: $363-369$.

18. DeLong ER, DeLong DM, Clarke-Pearson DL. Comparing the areas under two or more correlated receiver operating characteristic curves: A nonparametric approach. Biometrics 1988; 44: 837-845.

19. Pencina MJ, D'Agostino RB Sr, D'Agostino RB Jr, Vasan RS Evaluating the added predictive ability of a new marker: From area under the ROC curve to reclassification and beyond. Stat Med 2008; 27: $157-172$.

20. deFilippi C, Wasserman S, Rosanio S, Tiblier E, Sperger H, Tocchi $\mathrm{M}$, et al. Cardiac troponin $\mathrm{T}$ and $\mathrm{C}$-reactive protein for predicting prognosis, coronary atherosclerosis, and cardiomyopathy in patients undergoing long-term hemodialysis. JAMA 2003; 290: 353-359.

21. Satyan S, Light RP, Agarwal R. Relationships of N-terminal pro-Bnatriuretic peptide and cardiac troponin $\mathrm{T}$ to left ventricular mass and function and mortality in asymptomatic hemodialysis patients. Am J Kidney Dis 2007; 50: 1009-1019.

22. Mallamaci F, Tripepi G, Cutrupi S, Malatino LS, Zoccali C. Prognostic value of combined use of biomarkers of inflammation, endothelial dysfunction, and myocardiopathy in patients with ESRD. Kidney Int 2005; 67: 2330-2337.

23. Wang AY, Lam CW, Wang M, Chan IH, Goggins WB, Yu CM, et al. Prognostic value of cardiac troponin T is independent of inflammation, residual renal function, and cardiac hypertrophy and dysfunction in peritoneal dialysis patients. Clin Chem 2007; 53: $882-$ 889.

24. Apple FS, Murakami MM, Pearce LA, Herzog CA. Multi-biomarker risk stratification of $\mathrm{N}$-terminal pro-B-type natriuretic peptide, high-sensitivity $\mathrm{C}$-reactive protein, and cardiac troponin $\mathrm{T}$ and $\mathrm{I}$ in end-stage renal disease for all-cause death. Clin Chem 2004; 50: 2279-2285.

25. Bargnoux AS, Morena M, Jaussent I, Maurice F, Chalabi L, LerayMoragues $\mathrm{H}$, et al. A combined index of cardiac biomarkers as a risk factor for early cardiovascular mortality in hemodialysis patients. Clin Chem Lab Med 2013; 51: 1865-1874.

26. Hallén J, Madsen L, Ladefoged S, Fagerland MW, Serebruany VL, Agewall S, et al. Incremental value of a combination of cardiac troponin $\mathrm{T}, \mathrm{N}$-terminal pro-brain natriuretic peptide and $\mathrm{C}$-reactive protein for prediction of mortality in end-stage renal disease. Scand J Urol Nephrol 2011; 45: 151-158.

27. Geerse DA, van Berkel M, Vogels S, Kooman JP, Konings CJ, Scharnhorst V. Moderate elevations of high-sensitivity cardiac troponin I and B-type natriuretic peptide in chronic hemodialysis patients are associated with mortality. Clin Chem Lab Med 2013; 51: $1321-1328$.

28. Hickman PE, McGill DA, Talaulikar G, Hiremagalur B, Bromley J,
Rahman A, et al. Prognostic efficacy of cardiac biomarkers for mortality in dialysis patients. Intern Med J 2009; 39: 812-818.

29. McGill D, Talaulikar G, Potter JM, Koerbin G, Hickman PE. Over time, high-sensitivity TnT replaces NT-proBNP as the most powerful predictor of death in patients with dialysis-dependent chronic renal failure. Clin Chim Acta 2010; 411: 936-939.

30. London GM. Cardiovascular disease in chronic renal failure: Pathophysiologic aspects. Semin Dial 2003; 16: 85-94.

31. Damman P, Beijk MA, Kuijt WJ, Verouden NJ, van Geloven N, Henriques JP, et al. Multiple biomarkers at admission significantly improve the prediction of mortality in patients undergoing primary percutaneous coronary intervention for acute ST-segment elevation myocardial infarction. J Am Coll Cardiol 2011; 57: 29-36.

32. Thygesen K, Mair J, Giannitsis E, Mueller C, Lindahl B, Blankenberg $\mathrm{S}$, et al; Study Group on Biomarkers in Cardiology of the ESC Working Group on Acute Cardiac Care. How to use high-sensitivity cardiac troponins in acute cardiac care. Eur Heart J 2012; 33: $2252-$ 2257.

33. Apple FS, Collinson PO; IFCC Task Force on Clinical Applications of Cardiac Biomarkers. Analytical characteristics of high-sensitivity cardiac troponin assays. Clin Chem 2012; 58: 54-61.

34. Mueller M, Vafaie M, Biener M, Giannitsis E, Katus HA. Cardiac troponin T: From diagnosis of myocardial infarction to cardiovascular risk prediction. Circ J 2013; 77: 1653-1661.

35. Held PJ, Brunner F, Odaka M, Garcia JR, Port FK, Gaylin DS. Fiveyear survival for end-stage renal disease patients in the United States, Europe, and Japan, 1982 to 1987. Am J Kidney Dis 1990; 15: 451457.

36. Sekikawa A, Ueshima H, Kadowaki T, El-Saed A, Okamura T, Takamiya $\mathrm{T}$, et al. Less subclinical atherosclerosis in Japanese men in Japan than in White men in the United States in the post-World War II birth cohort. Am J Epidemiol 2007; 165: 617-624.

37. Kumada Y, Ishii H, Aoyama T, Kamoi D, Kawamura Y, Sakakibara $\mathrm{T}$, et al. Long-term clinical outcome after surgical or percutaneous coronary revascularization in hemodialysis patients. Circ J 2014; 78: 986-992.

38. Coe CL, Love GD, Karasawa M, Kawakami N, Kitayama S, Markus $\mathrm{HR}$, et al. Population differences in proinflammatory biology: Japanese have healthier profiles than Americans. Brain Behav Immun 2011; 25: 494-502.

39. Oldroyd JC, Heald A, Bansal N, Vyas A, Siddals K, Gibson M, et al. Inflammatory markers and growth in South Asian and European origin infants in Britain: The Manchester Children's Growth and Vascular Health Study. Atherosclerosis 2009; 207: 227-231.

40. K/DOQI Workgroup. KDOQI clinical practice guidelines for cardiovascular disease in dialysis patients. Am J Kidney Dis 2005; 45(Suppl 3): $\mathrm{S} 1-\mathrm{S} 153$. 